ANNALES

POLONICI MATHEMATICI

XLVIII (1988)

\title{
On the Chern character of glued bundles
}

\author{
by JAN A. Rempata (Warszawa)
}

\begin{abstract}
We give an expression for the differential form representing the Chern character of a bundle obtained by glueing together two vector bundles (one of which is trivial) in terms of the glueing isomorphism.
\end{abstract}

Let $M$ be a $C^{\infty}$-smooth paracompact manifold and let $M_{1}, M_{2}$ be its open subsets with

$$
M_{1} \cup M_{2}=M, \quad M_{1} \cap M_{2}=M_{12} .
$$

Let $P_{1}$ and $P_{2}$ be smooth complex vector bundles over $M_{1}$ and $M_{2}$ respectively (smoothly), isomorphic over $M_{12}$. It is well known that there exists a vector bundle $P$ over $M$ with $P \mid M_{i} \cong P_{i}, i=1,2$, and any two such bundles are isomorphic.

Following (Fedosov $\left({ }^{1}\right)$ ) we shall assume that $P_{i}$ is defined by a smooth family of projectors $P_{i}(x): C^{N} \rightarrow C^{N}, x \in M_{i}$. Then any mutually inverse isomorphisms $a: P_{1}\left|M_{12} \rightarrow P_{2}\right| M_{12}$ and $b: P_{2}\left|M_{12} \rightarrow P_{1}\right| M_{12}$ can be realized by smooth families of linear maps $a(x), b(x): C^{N} \rightarrow C^{n}, x \in M_{12}$, satisfying the relations

$$
\begin{aligned}
a(x) P_{1}(x) & =P_{2}(x) a(x)=a(x), \\
b(x) P_{2}(x) & =P_{1}(x) b(x)=b(x), \\
b(x) a(x) & =P_{1}(x), \\
a(x) b(x) & =P_{2}(x) .
\end{aligned}
$$

Let $f_{1}, f_{2}$ be smooth real-valued functions subject to the conditions

$$
\operatorname{supp} f_{i} \subset M_{1}, \quad f_{1}^{2}+f_{2}^{2}=1 .
$$

Then the bundle $P$ may be defined by the projectors

$$
\begin{gathered}
P(x): C^{N} \times C^{N} \rightarrow C^{N} \times C^{N}, \quad x \in M, \\
P(x)=\left[\begin{array}{ll}
f_{1}^{2}(x) P_{1}(x) & f_{1}(x) f_{2}(x) b(x) \\
f_{1}(x) f_{2}(x) a(x) & f_{2}^{2}(x) P_{2}(x)
\end{array}\right] .
\end{gathered}
$$

(') B. V. Fedosov, On the index of an elliptic system on a manifold (in Russian), Funktsional. Anal. Prilozhen. 4 (4) (1970), 57-67. 
We wish to express the differential form $\operatorname{ch} P$ representing the Chern character of $P$ by forms derived directly from $P_{1}, P_{2}, a, b$ (see footnote $\left({ }^{1}\right)$ ). This is interesting in connection with the Atiyah-Singer index formula (Fedosov, footnote $\left({ }^{1}\right)$ ).

According to

$$
\operatorname{ch} P=\operatorname{dim} P+\sum_{k=1}\left(\frac{i}{2 \pi}\right)^{k} \frac{1}{k !} \operatorname{Tr} w_{p}^{k}
$$

where $w_{p}=P d P d P$ (for simplicity we omit the sign of exterior product) and $\operatorname{Tr} w_{p}^{k}$ denotes the trace of the $k$-th exterior power of $w_{p}$. Thus all we need is to calculate $\operatorname{Tr} w_{p}^{k}$.

We do this under the assumption (not very restrictive, in fact) that $P_{2}$ is a trivial bundle, i.e., that $x \mapsto P_{2}(x)$ is a constant function.

Setting

$$
\begin{gathered}
Q_{1}=\left[\begin{array}{ll}
d\left(f_{1}^{2}\right) P_{1} & d\left(f_{1} f_{2}\right) b \\
d\left(f_{1} f_{2}\right) a & d\left(f_{2}^{2}\right) P_{2}
\end{array}\right], \\
Q_{2}=\left[\begin{array}{cc}
f_{1}^{2} d P_{1} & f_{1} f_{2} d b \\
f_{1} f_{2} d a & 0
\end{array}\right],
\end{gathered}
$$

after straightforward calculations we obtain the following relations:

$$
\begin{aligned}
d P & =Q_{1}+Q_{2}, & & \\
P Q_{1}+Q_{1} P & =Q_{1}, & & \\
Q_{2}^{2 k+1} P & =Q_{2}^{2 k+1}-P Q_{2}^{2 k+1}, & & k=0,1, \ldots, \\
Q_{2}^{2 k} P & =P Q_{2}^{2 k}, & k & =1,2, \ldots,
\end{aligned}
$$

$R_{1} Q_{1} R_{2} Q_{1} R_{3}=0$ for all matrix-valued forms $R_{1}, R_{2}, R_{3}$,

$$
P Q_{1}-Q_{1} P=\left(f_{2} d f_{1}-f_{1} d f_{2}\right)\left[\begin{array}{rr}
0 & -b \\
a & 0
\end{array}\right]
$$

Using the above relations we get by induction

$$
\operatorname{Tr} w_{p}^{k}=\operatorname{Tr} Q_{2}^{2 k} P+k \operatorname{Tr}\left(P Q_{1}-Q_{1} P\right) Q_{2}^{2 k-1}, \quad k=1,2, \ldots
$$

Now, let $A_{l}, B_{l}, C_{l}, D_{l}$ be matrix-valued forms such that

$$
Q_{2}^{l}=\left[\begin{array}{ll}
A_{l} & C_{l} \\
B_{l} & D_{l}
\end{array}\right], \quad l=0,1, \ldots
$$


Since $Q_{2}^{l+1}=Q_{2} Q_{2}^{l}$ it is easy to get

$$
\begin{gathered}
A_{l+2}=f_{1}^{2} d P_{1} A_{l+1}+f_{1}^{2} f_{2}^{2} d b d a A_{l}, \\
A_{0}=E \quad \text { (the unit matrix), } \\
A_{1}=f_{1}^{2} d P_{1}, \\
B_{l+1}=f_{1} f_{2} d a A_{l}, \\
C_{l+2}=f_{1}^{2} d P_{1} C_{l+1}+f_{1}^{2} f_{2}^{2} d b d a C_{l}, \\
C_{0}=0, \quad C_{1}=f_{1} f_{2} d b, \\
D_{l+1}=f_{1} f_{2} d a C_{l} .
\end{gathered}
$$

By induction, from (3) and $\left(5^{\prime}\right)$ it easily follows that

$$
C_{l+1}=f_{1} f_{2} A_{l} d b, \quad l=0,1, \ldots,
$$

and thus

$$
D_{I+1}=f_{1}^{2} f_{2}^{2} d a A_{l-1} d b, \quad l=1,2, \ldots
$$

Define matrix-valued forms on $M_{12}$ by

$$
v_{0}=b d a, \quad v_{1}=d b a \text {. }
$$

Then we have $d P_{1} \mid M_{12}=v_{0}+v_{1}, d b d a=v_{1} v_{0}$. By (2) and (4)-(6)

$$
\begin{gathered}
\operatorname{Tr} Q_{2}^{2 k} P=f_{1}^{2} \operatorname{Tr} P_{1} A_{2 k}-f_{2}^{2} \operatorname{Tr} A_{2 k}+2 f_{1}^{2} f_{2}^{2} \operatorname{Tr} v_{0} A_{2 k-1}, \\
\operatorname{Tr}\left(P Q_{1}-Q_{1} P\right) Q_{2}^{2 k-1}=f_{1} d f_{1} \operatorname{Tr}\left(v_{1}-v_{0}\right) A_{2 k-2}
\end{gathered}
$$

and so (1) may be rewritten as

(7) $\quad \operatorname{Tr} w_{p}^{k}=f_{1}^{2} \operatorname{Tr} P_{1} A_{2 k}-f_{2}^{2} \operatorname{Tr} A_{2 k}+2 f_{1}^{2} f_{2}^{2} \operatorname{Tr} v_{0} A_{2 k-1}+$

$$
+k f_{1} d f_{1} \operatorname{Tr} v_{1} A_{2 k-2}-k f_{1} d f_{1} \operatorname{Tr} v_{0} A_{2 k-2} \text {. }
$$

Now we pass to the calculation of $A_{l}$.

Let $F_{l}$ be the set of all $l$-tuples consisting of 0 or 1 . For $\left(i_{l}, i_{l-1}, \ldots, i_{1}\right)$ $=I \in F_{l}$ denote by $n(I)$ the number of all indices $p, 2 \leqslant p \leqslant k$, such that $i_{p}=1$ and $i_{p-1}=0$.

The function $n(\cdot)$ has the following properties:'

$$
\begin{aligned}
n(\emptyset) & =0, \quad n(0)=n(1)=1, \\
n(0, I) & =n(I), \\
n(1,1, I) & =n(1, I), \\
n(1,0, I) & =n(0, I)+1=n(I)+1 .
\end{aligned}
$$


For $I=\left(i_{l}, i_{l-1}, \ldots, i_{1}\right) \in F_{l}$ we set

$$
v^{I}=v_{i_{l}} v_{i_{l-1}} \ldots v_{i_{1}}, \quad V_{l}(m)=\sum_{U \in F_{l} ; m(I)=m !} v^{I} .
$$

Obviously,

$$
\left(d P_{1} \mid M_{12}\right)^{l}=\left(v_{0}+v_{1}\right)^{l}=\sum_{m \geqslant 0} V_{l}(m)
$$

Lemma 1.

$$
A_{l}=f_{1}^{2 l}\left(d P_{1}\right)^{l}+\sum_{m \geqslant 1}\left(f_{1}^{2 l-2 m}-f_{1}^{2 l}\right) V_{l}(m)
$$

Proof. Let $A_{i}^{\prime}$ denote the right-hand side of the above formula. We shall show that $A_{i}^{\prime}$ satisfies (3).

Obviously, this is so for $l=0$ and $l=1$. Put

$$
F_{l}^{m}=\left\{I \in F_{l}: n(I)=m\right\}, \quad F_{l}^{*}=\left\{I \in F_{l}: n(I)>0\right\}
$$

and observe that in view of (8)

$$
F_{l+2}^{*}=\left\{(0, I): I \in F_{l+1}^{*}\right\} \cup\left\{(1,0, J): J \in F_{l}\right\} \cup\left\{(1,1, J):(1, J) \in F_{l+1}^{*}\right\} \text {. }
$$

We may write

and so, by (9),

$$
A_{i}^{\prime}=f_{1}^{2 l}\left(d P_{1}\right)^{l}+\sum_{I \in F_{i}^{*}}\left(f_{1}^{2 l-2 m(I)}-f_{1}^{2 l}\right) v^{I}
$$

Hence we have

$$
f_{2}^{2} A_{l}^{\prime}=\left(1-f_{1}^{2}\right) \sum_{I \in F_{l}} f_{1}^{2 l-2 \pi(I)} v^{I}
$$

$$
\begin{aligned}
f_{1}^{2} d P_{1} A_{i+1}^{\prime}+f_{1}^{2} f_{2}^{2} v_{0} A_{l}^{\prime} & \\
=f_{l}^{2 l+4}\left(d P_{1}\right)^{l+2} & +\sum_{J \in F_{i+1}^{*}}\left(f_{1}^{2 l+4-2 m(I)}-f_{1}^{2 l+4}\right)\left(v_{0}+v_{1}\right) v^{I}+ \\
& +\sum_{J \in F_{l}} f_{1}^{2 l+2-2 m(J)} v_{1} v_{0} v^{J}-\sum_{J \in F_{l}} f_{1}^{2 l+4-2 n(J)} v_{1} v_{0} v^{J} \\
=f_{1}^{2 l+4}\left(d P_{1}\right)^{l+2} & +\sum_{I \in F_{i+1}^{*}}\left(f_{1}^{2 l+4-2 m(0, I)}-f_{1}^{2 l+4}\right) v^{(0, I)}+ \\
& +\sum_{(1, J) \in F_{i+1}^{*}}\left(f_{1}^{2 l+4-2 m(1, J)}-f_{1}^{2 l+4}\right) v^{(1,1, J)}+ \\
& +\sum_{J \in F_{l}} f_{1}^{2 l+4-2 m(0, J)} v^{(1,0, J)}-\sum_{J \in F_{l}} f_{1}^{2 l+4} v^{(1,0, J)}+
\end{aligned}
$$




$$
\begin{aligned}
& +\sum_{J \in F_{l}} f_{1}^{2 l+2-2(n(1,0, J)-1)} v^{(1,0, J)}-\sum_{J \in F_{l}} f_{1}^{2 l+4-2 m(J)} v^{(1,0, J)} \\
=f_{1}^{2 l+4}\left(d P_{1}\right)^{l+2} & +\sum_{(0, I) \in F_{l+2}^{*}}\left(f_{1}^{2 l+4-2 n(0, I)}-f_{1}^{2 l+4}\right) v^{(0, I)}+ \\
& +\sum_{(1,1, J) \in F_{l+2}^{*}}\left(f_{1}^{2 l+4-2 n(1,1, J)}-f_{1}^{2 l+4}\right) v^{(1,1, J)}+ \\
& +\sum_{(1,0, J) \in F_{l+2}^{*}}\left(f_{1}^{2 l+4-2 n(1,0, J)}-f^{2 l+4}\right) v^{(1,0, J)} \\
=f_{1}^{2 l+4}\left(d P_{1}\right)^{l+2} & +\sum_{I \in F_{l+2}^{*}}\left(f_{1}^{2 l+4-n(I)}-f_{1}^{2 l+4}\right) v^{I}=A_{l+2}^{\prime} .
\end{aligned}
$$

This implies the assertion of the lemma.

Corollary.

$$
A_{l} \mid M_{12}=\sum_{m \geqslant 0} f_{1}^{2 l-2 m} V_{l}(m) .
$$

Now consider formula (7) for $\operatorname{Tr} w_{p}^{k}$.

Since the supports of the forms $d f_{1}, f_{1}^{2 k-2 m}-f_{1}^{2 k}(k \geqslant m \geqslant 1), f_{1} f_{2}$ are contained in $M_{12}$, we may use $\left(10^{\prime}\right)$. We obtain

$$
\begin{aligned}
\operatorname{Tr} w_{p}^{k}= & f_{1}^{4 k+2} \operatorname{Tr} P_{1}\left(d P_{1}\right)^{2 k}+ \\
& +\sum_{m \geqslant 1}\left(f_{1}^{4 k+2-2 m}-f_{1}^{4 k+2}\right) \operatorname{Tr} b a V_{2 k}(m)- \\
& -\sum_{m \geqslant 0} f_{2}^{2} f_{1}^{4 k-2 m} \operatorname{Tr} V_{2 k}(m)+2 \sum_{m \geqslant 0} f_{2}^{2} f_{1}^{4 k-2 m} \operatorname{Tr} v_{0} V_{2 k-1}(m)+ \\
& +k f_{1} d f_{1} \sum_{m \geqslant 0} f_{1}^{4 k-4-2 m}\left(\operatorname{Tr} v_{1} V_{2 k-2}(m)-\operatorname{Tr} v_{0} V_{2 k-2}(m)\right) .
\end{aligned}
$$

Let us define some new forms.

For positive integers $m, l, r_{1}, \ldots, r_{m}$ such that $r_{1}+\ldots+r_{m}=[l / 2]$ we put

$$
S_{l}\left(r_{1}, \ldots, r_{m}\right)=\operatorname{Tr} v_{1} v_{0}^{2 r_{1}-1} v_{1} v_{0}^{2 r_{2}-1} \ldots v_{1} v_{0}^{2 r_{m}-1-1} v_{1} v_{0}^{2 r_{m}-\mathrm{e}(t)}
$$

where

$$
\begin{gathered}
e(l)= \begin{cases}1 & \text { for } l \text { even, } \\
0 & \text { for } l \text { odd, }\end{cases} \\
T_{l}(m)=\sum_{r_{1}+\ldots+r_{m}=[l / 2]} r_{m} S\left(r_{1}, \ldots, r_{m}\right), \quad \tilde{T}_{l}(m)=\sum_{r_{1}+\ldots+r_{m}=[1 / 2]} S\left(r_{1}, \ldots, r_{m}\right) .
\end{gathered}
$$


Also, it is convenient to put

$$
\begin{aligned}
T_{l}(0) & =\operatorname{Tr} v_{0}^{l}, \\
\tilde{T}_{l}(0) & =0, \\
T_{l}(m) & =\tilde{T}_{l}(m)=0 \quad \text { for } m>[l / 2] \text { or } m<0, \\
Z_{k} & =\sum_{1 \leqslant m \leqslant k} \frac{k}{2 k-m} T_{2 k-1}(m-1) .
\end{aligned}
$$

The summands in formula (11) may be expressed by $T_{2 k}$. In the sequel we shall prove the following result.

LEMMA 2.

$$
\begin{aligned}
\operatorname{Tr} V_{2 k}(m)= & d T_{2 k-1}(m-1)-d T_{2 k-1}(m), \\
\operatorname{Tr} b a V_{2 k}(m)= & -\frac{k-m+1}{2 k-m+1} d T_{2 k-1}(m-2)+ \\
& +2 \frac{k-m}{2 k-m} d T_{2 k-1}(m-1)-d T_{2 k-1}(m), \\
\operatorname{Tr} v_{0} V_{2 k-1}(m)= & \frac{k-m}{2 k-m} d T_{2 k-1}(m-1)-d T_{2 k-1}(m), \\
\operatorname{Tr} v_{0} V_{2 k-2}(m)= & T_{2 k-1}(m)=-\operatorname{Tr} v_{1} V_{2 k-2}(m) .
\end{aligned}
$$

Substituting the formulas of Lemma 2 to (11), we find after simple reductions

$$
\begin{aligned}
\operatorname{Tr} w_{p}^{k}= & f_{1}^{4 k+2} \operatorname{Tr} w_{p_{1}}^{k}+\sum_{1 \leqslant m \leqslant k}\left(f_{1}^{4 k+2}-f_{1}^{4 k-2 m}\right) \frac{k}{2 k-m} d T_{2 k-1}(m-1)- \\
& -2 k f_{1} d f_{1} \sum_{1 \leqslant m \leqslant k} f_{1}^{4 k-2 m-2} T_{2 k-1}(m-1) \\
= & f_{1}^{4 k+2} \operatorname{Tr} w_{p_{1}}^{k}+\sum_{1 \leqslant m \leqslant k}\left(f_{1}^{4 k+2}-f_{1}^{4 k-2 m}\right) \frac{k}{2 k-m} d T_{2 k-1}(m-1)+ \\
& +\sum_{1 \leqslant m \leqslant k} d\left(f_{1}^{4 k+2}-f_{1}^{4 k-2 m}\right) \frac{k}{2 k-m} T_{2 k-1}(m-1)- \\
& -d f_{1}^{4 k+2} \sum_{1 \leqslant m \leqslant k} \frac{k}{2 k-m} T_{2 k-1}(m-1) \\
= & \operatorname{Tr} w_{p_{1}}^{k} f_{1}^{4 k+2}+Z_{k} d f_{1}^{4 k+2}+ \\
& +d\left(\sum_{1 \leqslant m \leqslant k}\left(f_{1}^{4 k+2}-f_{1}^{4 k-2 m}\right) \frac{k}{2 k-m} T_{2 k-1}(m-1)\right) .
\end{aligned}
$$


Since $\operatorname{supp}\left(f_{1}^{4 k+2}-f_{1}^{4 k-2 m}\right)$ is contained in $M_{12}$, the form

$$
Y_{k}=\sum_{1 \leqslant m \leqslant k}\left(f_{1}^{4 k+2}-f_{1}^{4 k-2 m}\right) \frac{k}{2 k-m} T_{2 k-1}(m-1)
$$

is well defined on all of $M$ and has support in $M_{12}$.

On the other hand, we have

$$
\begin{aligned}
\operatorname{Tr}\left(w_{p_{1}}^{k} \mid M_{12}\right) & =\operatorname{Tr} b a\left(v_{0}+v_{1}\right)^{2 k}=\sum_{0 \leqslant m \leqslant k} \operatorname{Tr} b a V_{2 k}(m) \\
& =-\sum_{1 \leqslant m \leqslant k} \frac{k}{2 k-m} d T_{2 k-1}(m-1)=-d Z_{k} .
\end{aligned}
$$

Now, let $g$ be any smooth function such that $g f_{1}=f_{1}$. Then $\operatorname{supp}\left(f_{1}^{4 k+2}-g\right)=\operatorname{supp} g\left(f_{1}^{4 k+2}-1\right)$ is contained in $M_{12}$ and thus

$$
Z_{k} d\left(f_{1}^{4 k+2}-g\right)+\operatorname{Tr} w_{p_{1}}^{k}\left(f_{2}^{4 k+2}-g\right)=-d\left(f_{1}^{4 k+2}-g\right) Z_{k}-\left(f_{1}^{4 k+2}-g\right) d Z_{k}
$$

where $Y_{k}^{\prime}=\left(f_{1}^{4 k+2}-g\right) Z_{k}$.

$$
=-d Y_{k}^{\prime},
$$

Finally,

$$
\operatorname{Tr} w_{p}^{k}=Z_{k} d g+g \operatorname{Tr} w_{p_{1}}^{k}+d\left(Y_{k}-Y_{k}^{\prime}\right)
$$

Consequently, $\operatorname{Tr} w_{p}^{k}$ is homologous to $Z_{k} d g+g \operatorname{Tr} w_{p_{1}}^{k}$, and the Chern character of $P$ can be represented by the form

$$
\operatorname{ch} P=g \operatorname{ch} P_{1}+Z d g, \quad \text { where } Z=\sum_{k=1}^{\infty}\left(\frac{i}{2 \pi}\right)^{k} \frac{1}{k !} Z_{k} .
$$

This is just the formula we wish to get.

If the bundle $P_{1}$ is trivial (see footnote $\left({ }^{1}\right)$ ), then $w_{p_{1}}=0$ and thus $\operatorname{ch} P$ $=Z d g$. Moreover, in this case $v_{1}=-v_{0}$ and so we can obtain a more explicit formula for $Z_{k}$ :

$$
\begin{aligned}
Z_{k} & =\sum_{1 \leqslant m \leqslant k} \frac{k}{2 k-m} \sum_{r_{1}+\ldots+r_{m-1}=k-1}(-1)^{m-1} r_{m-1} \operatorname{Tr} v_{0}^{2 k-1} \\
& =\sum_{1 \leqslant m \leqslant k}(-1)^{m-1}\left(\begin{array}{c}
k-1 \\
m-1
\end{array}\right) \frac{k}{2 k-m} \operatorname{Tr} v_{0}^{2 k-1} \\
& =(-1)^{k-1} \frac{k !(k-1) !}{(2 k-1) !} \operatorname{Tr}(b d a)^{2 k-1}
\end{aligned}
$$

This formula has been obtained by Fedosov (see footnote $\left(^{1}\right)$, p. 65).

To complete our calculation we have to prove Lemma 2. For this we need some auxiliary results.

First, we show that the forms $V_{l}(m)$ are closed. 
146

J. A. Rempała

Let us introduce the following forms on $M_{12}$ :

$$
\begin{aligned}
& u_{1}=d(b a), \quad u_{2}=v_{1} v_{0}, \\
& \tilde{U}_{l}(p)= \begin{cases}\sum_{a_{p}+\ldots+a_{1}=k} u_{a_{p}} u_{a_{p-1}} \ldots u_{a_{1}} & \text { for } l \geqslant 1,[(l+1) / 2] \leqslant p \leqslant l, \\
0 & \text { for } l<0 \text { or } p<[(l+1) / 2] \text { or } p>l, \\
E & \text { for } l=p=0,\end{cases} \\
& U_{l}(t)=\tilde{U}_{l}(l-t) .
\end{aligned}
$$

For $U_{1}(t)$ we have the inductive formula

$$
\begin{gathered}
U_{i}(t)=u_{1} U_{t-1}(t)+u_{2} U_{t-2}(t-1) \\
U_{1}(t)=0 \quad \text { for } l<0 \text { and all } t \\
U_{0}(0)=E, \quad U_{0}(t)=0 \quad \text { for all } t \neq 0 .
\end{gathered}
$$

Hence for $l \geqslant 1$

$$
U_{l}(t)=d\left(U_{l-1}^{*}(t)\right)
$$

where

$$
U_{l-1}^{*}(t)=b a U_{l-1}(t)+v_{0} U_{l-2}(t-1) .
$$

From (12) and (3) it follows by induction that

$$
A_{l} \mid M_{12}=\sum_{0 \leqslant t \leqslant[l / 2]} f_{1}^{2 t-2 t} f_{2}^{2 t} U_{l}(t) \text {. }
$$

Comparing the two formulas for $A_{l}$ we obtain a relation between $U_{l}$ and $V_{l}$.

LeMmA 3. The following equalities are valid on $M_{12}$ :

$$
\begin{gathered}
U_{l}(t)=\sum_{t \leqslant m \leqslant[l / 2]}\left(\begin{array}{l}
m \\
t
\end{array}\right) V_{l}(m), \\
V_{l}(m)=\sum_{m \leqslant t \leqslant[l / 2]}(-1)^{m+t}\left(\begin{array}{l}
t \\
m
\end{array}\right) U_{l}(t) .
\end{gathered}
$$

Proof.

$$
\begin{aligned}
\sum_{m \geqslant 0} f_{1}^{2 l-2 m} V_{l}(m) & =A_{l} \mid M_{12}=\sum_{0 \leqslant l \leqslant[l / 2]} f_{1}^{2 l-2 t}\left(1-f_{1}^{2}\right)^{t} U_{l}(t) \\
& =\sum_{0 \leqslant m \leqslant[l / 2]} f_{1}^{2 l-2 m} \sum_{m \leqslant t \leqslant[l / 2]}(-1)^{t+m}\left(\begin{array}{l}
t \\
m
\end{array}\right) U_{l}(t) .
\end{aligned}
$$


In a small neighbourhood of any given point $x \in M_{12}$ the function $f_{1}$ may be chosen arbitrarily. Thus in the above equality the coefficients at the same powers of $f_{1}$ must be equal. This proves the first assertion of lemma.

The second assertion follows directly from the first.

Corollary. $d V_{l}(m)=0$.

Proof.

$$
d V_{l}(m)=\sum_{m \leqslant t \leqslant[H 2]}(-1)^{m+t}\left(\begin{array}{l}
t \\
m
\end{array}\right) d U_{1}(t)=0 .
$$

Remark. From (15) and (13) it follows easily that $V_{l}(m)=d V_{l}^{*}(m)$, where

$$
V_{l-1}^{*}(m)=b a V_{l-1}(m)-v_{0} V_{l-2}(m)+v_{0} V_{l-2}(m-1) .
$$

LeMma 4.

$$
\begin{aligned}
\operatorname{Tr} V_{l}(m) & =\left(2 T_{l}(m)-\tilde{T}_{l}(m)-2 T_{l}(m+1)+\tilde{T}_{l}(m+1)\right) e(l), \\
\operatorname{Tr} b a V_{l}(m) & =\left(-T_{l}(m-1)+\tilde{T}_{l}(m-1)+2 T_{l}(m)-\right. \\
\left.-2 \tilde{T}_{l}(m)-2 T_{l}(m+1)+\tilde{T}_{l}(m+1)\right) e(1), & \\
\operatorname{Tr} v_{0} V_{l-1}(m) & =T_{l}(m)-\left(\tilde{T}_{l}(m)+2 T_{l}(m+1)-\tilde{T}_{l}(m+1)\right) e(l) \\
& =-\operatorname{Tr} v_{1} V_{l-1}(m), \\
\operatorname{Tr} v_{1} v_{0} V_{l-2}(m) & =\left(2 T_{2 k}(m+1)-\tilde{T}_{2 k}(m+1)\right) e(l) .
\end{aligned}
$$

All these formulas hold for $m \geqslant 0$.

Proof. Write $V\left(\begin{array}{l}p \\ q\end{array}\right)=v_{1}^{p} v g$ for $p, q \geqslant 0$. Then

$$
\begin{aligned}
& V_{l}^{(1)}(m)=\sum V\left(\begin{array}{l}
0 \\
q_{0}
\end{array}\right) V\left(\begin{array}{l}
p_{1} \\
q_{1}
\end{array}\right) \ldots V\left(\begin{array}{c}
p_{m} \\
q_{m}
\end{array}\right) V\left(\begin{array}{c}
p_{m+1} \\
0
\end{array}\right), \\
& V_{l}^{(2)}(m)=\sum V\left(\begin{array}{l}
0 \\
q_{0}
\end{array}\right) V\left(\begin{array}{l}
p_{1} \\
q_{1}
\end{array}\right) \ldots V\left(\begin{array}{l}
p_{m} \\
q_{m}
\end{array}\right) \\
& V_{l}^{(3)}(m)=\sum V\left(\begin{array}{l}
p_{1} \\
q_{1}
\end{array}\right) \ldots V\left(\begin{array}{l}
p_{m} \\
q_{m}
\end{array}\right) V\left(\begin{array}{c}
p_{m+1} \\
0
\end{array}\right) \\
& V_{l}^{(4)}(m)=\sum V\left(\begin{array}{l}
p_{1} \\
q_{1}
\end{array}\right) \ldots V\left(\begin{array}{l}
p_{m} \\
q_{m}
\end{array}\right) .
\end{aligned}
$$

Here in each sum all $p_{i}, q_{i}$ are positive (integers) and their sum is equal to $l$. 
Writing $(i=1, \ldots, k)$

$$
\begin{gathered}
V_{l}^{(i 0)}(m)=\operatorname{Tr} V_{l}^{(i)}(m), \quad V_{l}^{(i 1)}(m)=\operatorname{Tr} b a V_{l}^{(i)}(m), \quad V_{l}^{(i 2)}(m)=\operatorname{Tr} v_{0} V_{l-1}^{(i)}(m), \\
V_{l}^{(i 3)}(m)=\operatorname{Tr} v_{1} V_{l-1}^{(i)}(m), \quad V_{l}^{(i 4)}(m)=\operatorname{Tr} v_{1} v_{0} V_{l-2}^{(i)}(m),
\end{gathered}
$$

we see that $\sum_{l=1}^{4} V_{l}^{(i j)}(m)$ for $j=0,1, \ldots, 4$ is equal to $\operatorname{Tr} V_{l}(m), \operatorname{Tr} b a V_{l}(m)$, $\operatorname{Tr} v_{0} V_{l-1}(m), \operatorname{Tr} v_{1} V_{l-1}(m)$ and $\operatorname{Tr} v_{1} v_{0} V_{l-1}(m)$ respectively.

To compute $V_{l}^{(i j)}(m)$ we use the following easy facts:

(a) $b a v_{0}=v_{0}, v_{1} b a=v_{1}, v_{0} b a=-b a v_{1}$.

(b) $V\left(\begin{array}{l}p \\ q\end{array}\right)=(-1)^{p-1} V\left(\begin{array}{c}1 \\ p+q-1\end{array}\right)$ for $p, q \geqslant 1$.

(c) $\operatorname{Tr} A B=(-1)^{\operatorname{deg} A \operatorname{deg} B} \operatorname{Tr} B A$ for all matrix-valued forms $A, B$. form

It is now easy to see that each $V_{l}^{(i j)}(m)$ can be written as a sum of the

$$
V_{l}^{(i j)}(m)=\sum_{p_{1}+\ldots+p_{\bar{m}}+q_{1}+\ldots+q_{\bar{m}}=l} c\left(p_{\bar{m}}, q_{\bar{m}}\right) \operatorname{Tr} V\left(\begin{array}{l}
p_{1} \\
q_{1}
\end{array}\right) \ldots V\left(\begin{array}{l}
p_{\bar{m}} \\
q_{\bar{m}}
\end{array}\right)
$$

with $\bar{m}$ equal to one of the numbers: $m-1, m, m+1$.

On account of (b) and the equality $\sum_{1 \leqslant p \leqslant s-1}(-1)^{p-1}=e(s)$ we have

$$
\begin{gathered}
V_{l}^{(i)}(m)=\sum_{p_{1}+\ldots+p_{\bar{m}}+q_{1}+\ldots+q_{\bar{m}}=l}(-1)^{p_{1}+\ldots+p_{\bar{m}}-\bar{m}} c\left(p_{\bar{m}}, q_{\bar{m}}\right) \times \\
\times \operatorname{Tr} V\left(\begin{array}{c}
1 \\
p_{1}+q_{1}-1
\end{array}\right) \ldots V\left(\begin{array}{c}
1 \\
p_{\bar{m}}+q_{\bar{m}-1}
\end{array}\right) \\
=\sum_{s_{1}+\ldots+s_{\bar{m}}=l} e\left(s_{1}\right) \ldots e\left(s_{\bar{m}-1}\right) c\left(s_{\bar{m}}\right) \operatorname{Tr} V\left(\begin{array}{c}
1 \\
s_{1}-1
\end{array}\right) \ldots V\left(\begin{array}{c}
1 \\
s_{\bar{m}-1}
\end{array}\right) \\
=\sum_{r_{1}+\ldots+r_{m}=[1 / 2]} c_{i j}\left(r_{\bar{m}}\right) S_{l}\left(r_{1}, \ldots, r_{\bar{m}}\right) .
\end{gathered}
$$

Here $c\left(p_{\bar{m}}, q_{\bar{m}}\right), c\left(s_{\bar{m}}\right), c_{i j}\left(r_{\bar{m}}\right)$ are some numbers.

For instance, for $i=1, j=0, m \geqslant 1$ we have

$$
\begin{aligned}
V_{l}^{(10)}(m) & =\sum_{\sum p_{i}+\sum q_{i}=l} \operatorname{Tr} V\left(\begin{array}{l}
0 \\
q_{0}
\end{array}\right) V\left(\begin{array}{l}
p_{1} \\
q_{1}
\end{array}\right) \ldots V\left(\begin{array}{l}
p_{m} \\
q_{m}
\end{array}\right) V\left(\begin{array}{c}
p_{m+1} \\
0
\end{array}\right) \\
& =\sum_{p+\sum p_{i}+q+\sum q_{i}=l}(-1)^{q(l+1)} \operatorname{Tr} V\left(\begin{array}{l}
p_{1} \\
q_{1}
\end{array}\right) \ldots V\left(\begin{array}{l}
p_{m} \\
q_{m}
\end{array}\right) V\left(\begin{array}{l}
p \\
q
\end{array}\right)
\end{aligned}
$$




$$
\begin{aligned}
& =\sum_{p+\sum p_{i}+q+\sum q_{i}=l}(-1)^{q l+q+p-1}(-1)^{p_{1}+\ldots+p_{m}-1} \times \\
& \times \operatorname{Tr} V\left(\begin{array}{c}
1 \\
p_{1}+q_{1}-1
\end{array}\right) \ldots V\left(\begin{array}{c}
1 \\
p+q-1
\end{array}\right) \\
& =\sum_{\substack{s_{1}+\ldots+s_{m+1}=l \\
p+q=s_{m+1}}}(-1)^{q l+s_{m+1}-1}(-1)^{p_{1}-1} \ldots(-1)^{p_{m}-1} \times \\
& \times \operatorname{Tr} V\left(\begin{array}{c}
1 \\
s_{1}-1
\end{array}\right) \ldots V\left(\begin{array}{c}
1 \\
s_{m+1}-1
\end{array}\right) \\
& =\sum_{\substack{s_{1}+\ldots+s_{m+1}=l \\
p+q=s_{m+1}}}(-1)^{q l+s_{m+1}-1} e\left(s_{1}\right) \ldots e\left(s_{m}\right) \operatorname{Tr} V\left(\begin{array}{c}
1 \\
s_{1}-1
\end{array}\right) \ldots V\left(\begin{array}{c}
1 \\
s_{m+1}-1
\end{array}\right) \\
& =\sum_{\substack{r_{1}+\ldots+r_{m+1}=[l / 2] \\
p+q=2 r_{m+1}+1-e(l)}}(-1)^{q l+2 r_{m+1}+e(l)} \operatorname{Tr} V\left(\begin{array}{c}
1 \\
2 r_{1}-1
\end{array}\right) \ldots V\left(\begin{array}{c}
1 \\
2 r_{m+1}-e(l)
\end{array}\right) \\
& =\sum_{\substack{\left.r_{1}+\ldots+r_{m+1}=[l] 2\right] \\
1 \leqslant q \leqslant 2 r_{m+1}-e(l)}}(-1)^{q l+1+1} S_{l}\left(r_{1}, \ldots, r_{m+1}\right) \\
& =\sum_{r_{1}+\ldots+r_{m+1}=[l / 2]}\left(1-2 r_{m+1}\right) e(l) S_{l}\left(r_{1}, \ldots, r_{m+1}\right) \text {. }
\end{aligned}
$$

Thus

$$
c_{10}\left(r_{m}\right)=c_{10}\left(r_{m+1}\right)=\left(1-2 r_{m+1}\right) e(l)
$$

and

$$
\begin{aligned}
V_{l}^{(10)}(m) & =e(l) \sum_{r_{1}+\ldots+r_{m+1}=[l / 2]} S_{l}\left(r_{1}, \ldots, r_{m+1}\right)- \\
& -2 e(l) \sum_{r_{1}+\ldots+r_{m+1}=[l / 2]} r_{m+1} S_{l}\left(r_{1} \ldots r_{m+1}\right) \\
& =\left(\tilde{T}_{l}(m+1)-2 T_{l}(m+1)\right) e(l) .
\end{aligned}
$$

In a similar way we obtain (for $m \geqslant 1$ )

$$
\begin{aligned}
c_{11} & =c_{10}=\left(1-2 r_{m+1}\right) e(l), \\
V_{l}^{(1,1)}(m) & =V_{l}^{(10)}(m)=\left(\tilde{T}_{l}(m+1)-2 T_{l}(m+1)\right) e(l), \\
c_{12} & =\sum_{p+q=2 r_{m+1}-e(l)}(-1)^{(q+1)(l+1)+p-1}=1+\left(1-2 r_{m+1}\right) e(l), \\
V_{l}^{(12)}(m) & =\tilde{T}_{l}(m+1)+\left(\tilde{T}_{l}(m+1)-2 T_{l}(m+1)\right) e(l),
\end{aligned}
$$




$$
\begin{aligned}
& c_{13}=\sum_{p+q=2 r_{m+1}-e(l)}(-1)^{(l+1)(q+1)+p}=-1+\left(2 r_{m+1}-1\right) e(l), \\
& V_{l}^{(13)}(m)=-\tilde{T}_{l}(m+1)+\left(2 T_{l}(m+1)-\tilde{T}_{l}(m+1)\right) e(l), \\
& c_{20}=c_{21}=\sum_{p+q+1=2 r_{m}+1-e(l)}(-1)^{q(l+1)+p-1}=r_{m}-e(l), \\
& V_{l}^{(20)}(m)=T_{l}(m)-\tilde{T}_{l}(m) e(l)=V_{l}^{(21)}(m) \text {, } \\
& c_{22}=\sum_{p+q+s=2 r_{m}-e(l)}(-1)^{(q+1)(1+1)+p-1}=r_{m}-1 \text {, } \\
& V_{i}^{(22)}(m)=T_{l}(m)-\tilde{T}_{i}(m) \text {, } \\
& c_{23}=\sum_{q=2 r_{m+1}-e(l)}(-1)^{(q+1)(l+1)}=1, \\
& V_{l}^{(23)}(m)=\tilde{T}_{l}(m+1) \text {, } \\
& c_{30}=c_{31}=\sum_{p+q+s=2 r_{m}+1-e(1)}(-1)^{(p+q)(l+1)+p+s-1} \\
& =-r_{m}+\left(2 r_{m}-1\right) e(l) \text {, } \\
& V_{l}^{(30)}(m)=V_{l}^{(31)}(m)=-T_{l}(m)+\left(2 T_{l}(m)-\tilde{T}_{l}(m)\right) e(l), \\
& c_{32}=\sum_{p=2 r_{m+1}-e(1)}(-1)^{1+p}=-1, \\
& V_{l}^{(32)}(m)=-\tilde{T}_{l}(m+1) \text {, } \\
& c_{33}=\sum_{p+q+s=2 r_{m}-e(t)}(-1)^{(p+q+1)(l+1)+p+s}=1-r_{m}, \\
& V_{l}^{(33)}(m)=\tilde{T}_{l}(m)-T_{l}(m), \\
& c_{40}=\sum_{p+q=2 r_{m}+1-e(l)}(-1)^{p-1}=e(l), \\
& V_{l}^{(40)}(m)=e(l) \tilde{T}_{l}(m) \text {, } \\
& c_{41}=\sum_{p+q+s+1=2 r_{m-1}+1-e(l)}(-1)^{(p+q)(t+1)+p+s-1}=\left(1-r_{m-1}\right) e(l), \\
& V_{l}^{(41)}(m)=\left(\tilde{T}_{l}(m-1)-T_{l}(m-1)\right) e(l) \text {, } \\
& c_{42}=\sum_{p+q=2 r_{m}-e(l)}(-1)^{l+p}=1-e(l), \\
& V_{l}^{(42)}(m)=\tilde{T}_{l}(m)-e(l) \tilde{T}_{l}(m), \\
& c_{43}=\sum_{p+q=2 r_{m}-e(l)}(-1)^{(p+q+1)(l+1)+p}=e(l)-1, \\
& V_{l}^{(43)}(m)=e(l) \tilde{T}_{l}(m)-\tilde{T}_{l}(m) .
\end{aligned}
$$


Summing up the suitable terms we get the first, second and third formulas of Lemma 4 for $m \geqslant 1$. For $m=0$ the calculation needs slight modifications but is very simple. Also, the calculation of $\operatorname{Tr} v_{1} v_{0} V_{i-2}$ can be done in a very similar way, but now new forms appear:

$$
T_{i}^{\prime}(m)=\sum_{r_{1}+\ldots+r_{m-1}=[l / 2]-1} S_{l}\left(r_{1}, \ldots, r_{m-1}, 1\right) .
$$

We get

$$
\begin{aligned}
& V_{l}^{(14)}(m)=e(l)\left(2 T_{l}(m+1)-3 T_{l}(m+1)+T_{l}^{\prime}(m+1)\right), \\
& V_{l}^{(24)}(m)=\tilde{T}_{l}(m+1)-T_{l}^{\prime}(m+1), \\
& V_{l}^{(34)}(m)=\left(\tilde{T}_{l}(m+1)-T_{l}^{\prime}(m+1)\right)(2 e(l)-1) \\
& V_{l}^{(44)}(m)=e(l) T_{l}^{\prime}(m+1)
\end{aligned}
$$

and thus

$\operatorname{Tr} v_{1} v_{0} V_{l-2}(m)=V_{l}^{(14)}(m)+\ldots+V_{l}^{(44)}(m)=e(l)\left(2 T_{l}(m+1)-\tilde{T}_{l}(m+1)\right)$ as asserted.

Lemma 5. For all $m \geqslant 0, k \geqslant 0$

$$
\tilde{T}_{2 k}(m)=\frac{m}{k} T_{2 k}(m) .
$$

Proof. It is easy to see that for any fixed $j, 1 \leqslant j \leqslant m$,

$$
\begin{aligned}
T_{2 k}(m) & =\sum_{r_{1}+\ldots+r_{m}=k} r_{m} S_{2 k}\left(r_{1}, \ldots, r_{m}\right) \\
& =\sum_{r_{1}+\ldots+r_{m}=k} r_{j} S_{2 k}\left(r_{1}, \ldots, r_{m}\right) .
\end{aligned}
$$

Thus

$$
\begin{aligned}
k \tilde{T}_{2 k}(m) & =\sum_{r_{1}+\ldots+r_{m}=k} k S_{2 k}\left(r_{1}, \ldots, r_{m}\right) \\
& =\sum_{r_{1}+\ldots+r_{m}=k}\left(r_{1}+\ldots+r_{m}\right) S_{2 k}\left(r_{1}, \ldots, r_{m}\right) \\
& =m \sum_{r_{1}+\ldots+r_{m}=k} r_{m} S_{2 k}\left(r_{1}, \ldots, r_{m}\right)=m T_{2 k}(m)
\end{aligned}
$$

as asserted.

LeMMA 6.

$$
\begin{aligned}
d T_{2 k}(m) & =0 \\
d T_{2 k-1}(m) & =2 T_{2 k}(m+1)-\tilde{T}_{2 k}(m+1)=\frac{2 k-m-1}{k} T_{2 k}(m+1) .
\end{aligned}
$$


Proof. Since $d v_{1}=-v_{1} v_{0}=-d v_{0}$, a simple computation gives $d V\left(\begin{array}{l}1 \\ 1\end{array}\right)$ $=0$ and for $r>1$

$$
\begin{aligned}
d V\left(\begin{array}{c}
1 \\
2 r-1
\end{array}\right) & =\sum_{\substack{s+t=2 r-1 \\
s, t \geqslant 1}}(-1)^{s+1} V\left(\begin{array}{l}
1 \\
s
\end{array}\right) V\left(\begin{array}{l}
1 \\
t
\end{array}\right) \\
& =\sum_{\substack{p+q=r \\
p, q \geqslant 1}}\left(V\left(\begin{array}{c}
1 \\
2 p-1
\end{array}\right) V\left(\begin{array}{l}
1 \\
2 q
\end{array}\right)-V\left(\begin{array}{c}
1 \\
2 p
\end{array}\right) V\left(\begin{array}{c}
1 \\
2 q-1
\end{array}\right)\right) .
\end{aligned}
$$

Using this, it is easy to get $d \tilde{T}_{2 k}(m)=0$ and thus by Lemma $5, d T_{2 k}(m)=0$.

The second assertion follows from Lemmas 4 and 3 . We have $T_{2 k-1}(m)$ $=\operatorname{Tr} v_{0} V_{2 k-2}(m)$ and hence

$$
\begin{aligned}
d T_{2 k-1}(m) & =\operatorname{Tr} d v_{0} V_{2 k-2}(m)-\operatorname{Tr} v_{0} d V_{2 k-2}(m)=\operatorname{Tr} v_{1} v_{0} V_{2 k-2}(m) \\
& =2 T_{2 k}(m+1)-\widetilde{T}_{2 k}(m+1)=\frac{2 k-m-1}{k} T_{2 k}(m+1) .
\end{aligned}
$$

Now we can give the proof of Lemma 2. By Lemmas 4, 5 and 6 we have

$$
\begin{aligned}
\operatorname{Tr} V_{2 k}(m)= & 2 T_{2 k}(m)-\tilde{T}_{2 k}(m)-\left(2 T_{2 k}(m+1)-\tilde{T}_{2 k}(m+1)\right) \\
= & d T_{2 k-1}(m-1)-d T_{2 k-1}(m),
\end{aligned} \quad \begin{gathered}
\operatorname{Tr} b a V_{2 k}(m)=-\frac{k-m+1}{k} T_{2 k}(m-1)+ \\
\quad+2 \frac{k-m}{k} T_{2 k}(m)-\frac{2 k-m-1}{k} T_{2 k}(m+1) \\
=-\frac{k-m+1}{2 k-m+1} d T_{2 k-1}(m-2)+ \\
\quad+2 \frac{k-m}{2 k-m} d T_{2 k-1}(m-1)-d T_{2 k-1}(m), \\
\operatorname{Tr} v_{0} V_{2 k-1}(m)=\frac{k-m}{k} T_{2 k}(m)-\frac{2 k-m-1}{k} T_{2 k}(m+1) \\
=\frac{k-m}{2 k-m} d T_{2 k-1}(m-1)-d T_{2 k-1}(m),
\end{gathered}
$$

$\operatorname{Tr} v_{0} V_{2 k-2}(m)=-\operatorname{Tr} v_{1} V_{2 k-2}(m)=T_{2 k-1}(m)$.

The proof is complete. 